gr-qc/9809030

\title{
The quadratic spinor Lagrangian is equivalent to the teleparallel theory
}

\author{
Roh Suan Tung * \\ Department of Physics, Lancaster University \\ Lancaster LA1 $4 Y B$, United Kingdom \\ James M. Nester ${ }^{\dagger}$ \\ Department of Physics and Center for Complex systems, National Central University \\ Chungli 320, Taiwan, R.O.C.
}

(23 March 1999)

\begin{abstract}
The quadratic spinor Lagrangian is shown to be equivalent to the teleparallel/tetrad representation of Einstein's theory. An important consequence is that the energy-momentum density obtained from this quadratic spinor Lagrangian is essentially the same as the "tensor" proposed by Møller in 1961.
\end{abstract}

PACS number(s): 04.20 Cv, 04.20 Fy

*Electronic address : r.tung@lancaster.ac.uk

$\dagger$ Electronic address : nester@joule.phy.ncu.edu.tw 


\section{INTRODUCTION}

The Quadratic Spinor Lagrangian (QSL) formulation of General Relativity (GR) [1 5 has an appeal that goes beyond aesthetics. One of its most promising features concerns gravitational energy-momentum and its localization.

Identifying a suitable energy-momentum expression for the gravitational field has long been an outstanding problem. The usual approaches lead to reference frame dependent pseudotensors; it seemed that the best one could get was a quasilocal expression. In contrast, in addition to links with the Witten type spinor formulation and an associated positive energy proof, the QSL seems to yield a covariant energy-momentum density [1]. This apparent covariance is here shown to be actually only cosmetic.

In the earlier investigations the role of the spinor field used in this formulation had not been clarified. In this present work it is shown that, at least for a large class of QSLs which we have considered, the spinor field is entirely an extra gauge field, which simply serves to give an attractive appearance to the formulas.

Using a certain particularly simple QSL, (with the aid of a suitable gauge) we show that the whole formulation is equivalent to the teleparallel (tetrad) reformulation of GR used by Møller in 1961 [6]; consequently the associated energy-momentum density coincides with the energy-momentum "tensor" found by Møller. This object is a tensor with regard to coordinate transformations but is not a tensor with regard to local Lorentz rotations of the frame. Hence the associated energy-momentum localization depends on a choice of Lorentz gauge. Thus we conclude that this QSL gives an energy-momentum density which actually depends on a choice of Lorentz gauge.

Recall that for Einstein's General Relativity there are various representations, these include (i) the metric, using coordinate frames, (ii) orthonormal frames, (iii) a teleparallel geometry, and (iv) the quadratic spinor formulation. Each representation reveals some insight and has some utility. We will briefly consider their relationships and compare the tensorial nature of their associated energy-momentum expressions. It turns out that the last three representations are essentially equivalent.

The Hilbert Lagrangian density for $\mathrm{GR}$ is $\mathcal{L}_{H}=-\sqrt{-g} R$. The traditional approach uses the metric coefficients in a coordinate basis as the dynamic variables, so $\mathcal{L}_{H}=\mathcal{L}_{H}(g, \partial g, \partial \partial g)$. Because of the second derivatives, this is not suitable for getting an energy-momentum density. However a certain (noncovariant) divergence can be removed (without affecting the equations of motion [6]) leading to Einstein's Lagrangian $\mathcal{L}_{E}=\mathcal{L}_{E}(g, \partial g)=\mathcal{L}_{H}-$ div. One can now apply the standard procedure and get the canonical energy-momentum density. It is known as the Einstein pseudotensor; its value depends to a large extent on the coordinate ("gauge") choice. No satisfying technique has been found to separate the "physics" from the coordinate gauge.

\section{MØLLER'S TETRAD/TELEPARALLEL REPRESENTATION}

An alternative is to use an orthonormal frame (tetrad), a pioneer of this approach was Møller [6]. Let $g_{\mu \nu}=g_{a b} e^{a}{ }_{\mu} e^{b}{ }_{\nu}$, with $g_{a b}=\operatorname{diag}(+1,-1,-1,-1)$, and regard the EinsteinHilbert Lagrangian as a function $\mathcal{L}_{e}(e, \partial e, \partial \partial e)$ of the tetrad $e^{a}{ }_{\mu}$. A suitable total divergence can again be removed yielding 


$$
\mathcal{L}_{M}=\mathcal{L}_{M}(e, \partial e)=\mathcal{L}_{e}-\operatorname{div}
$$

a Lagrangian density which is first order in the derivatives of the frame. Now the standard canonical energy-momentum density

$$
\sqrt{-g} T^{\mu}{ }_{\nu}=\frac{\partial \mathcal{L}_{M}}{\partial \partial_{\mu} e_{\lambda}^{a}} \partial_{\nu} e_{\lambda}^{a}-\delta_{\nu}^{\mu} \mathcal{L}_{M}
$$

is a tensor (density) under coordinate transformations, but it depends on the choice of orthonormal frame (Lorentz gauge). In other words it is not "tensorial" with respect to local "rotations" of the frame. An alternate geometric viewpoint of this situation is to use a teleparallel formulation.

Geometry includes, in general, the idea of parallel, which is determined by a connection. A priori the connection could be independent of the metric. Riemannian geometry (the standard type for GR) has a symmetric, metric compatible connection. Parallel transport is then determined entirely by the metric. One alternative is a teleparallel geometry (aka absolute parallel or Weitzenböck geometry [8]) which has a connection with vanishing curvature. Parallel transport is then path independent.

The tetrad formulation of GR can be represented in terms of a teleparallel geometry. This leads to the standard Teleparallel Equivalent of GR, which has been referred to by several names including GRtele, $\mathrm{GR}_{\|}$and TEGR. (For further discussion of this theory and its applications see [6,9 17] and the references contained therein.) The idea is to introduce a new parallel transport law. This can be done via a simple construction: (i) choose any orthonormal frame field, (ii) define it to be parallel. Then in this special OT (ortho-teleparallel) frame the connection coefficients vanish:

$$
\Gamma_{b \mu}^{a}:=\left(\nabla_{\mu} e_{b}\right)^{a}=0 ;
$$

consequently the curvature vanishes in this and every other frame. However the geometry is not trivial, for the components of the torsion tensor,

$$
T_{\mu \nu}^{a}=\partial_{\mu} e^{a}{ }_{\nu}-\partial_{\nu} e^{a}{ }_{\mu}+\Gamma_{b \mu}^{a} e_{\nu}^{b}-\Gamma_{b \nu}^{a} e_{\mu}^{b},
$$

in the OT frame reduce to $\partial_{\mu} e^{a}{ }_{\nu}-\partial_{\nu} e^{a}{ }_{\mu}$, which is generally nonvanishing. Being a tensor, the torsion will also be non-vanishing in any other reference frame.

Using this type of geometry, Einstein's GR theory can be obtained from a Lagrangian quadratic in torsion:

$$
\mathcal{L}_{T}=\sqrt{-g}\left(\frac{1}{4} T^{\alpha}{ }_{\mu \nu} T_{\alpha}{ }^{\mu \nu}+\frac{1}{2} T^{\alpha \beta \mu} T_{\beta \alpha \mu}-T_{\alpha \mu}^{\alpha} T_{\beta}^{\beta}{ }^{\mu}\right) .
$$

The solutions to the field equations and the associated energy-momentum tensor are now (teleparallel) gauge dependent. Hence the physics is represented by a whole gauge equivalence class of teleparallel geometries [18]. This can be regarded just as a (sometimes quite useful) geometric reformulation of the usual tetrad formulation of GR. The Lagrangian $\mathcal{L}_{T}$ is then just an alternate "more geometric" interpretation of $\mathcal{L}_{M}$. However, a strong case has been made for regarding this formulation as much more fundamental: seeing it as a gauge theory for local translations, and in fact the "correct" way to understand GR as a gauge theory [9,13] 16$]$. 


\section{THE QUADRATIC SPINOR LAGRANGIAN}

A few years ago (using some spinor-curvature identities [19]) we found some quadratic spinor actions for GR [1]. One of the simplest (recently we learned that a Lagrangian of the same form was used long ago for anticommuting Majorana spinors in the context of supergravity [20]) is dependent on a spinor valued one form $\Psi$ :

$$
S\left[\Psi, \omega^{a b}\right]=\int \mathcal{L}_{\Psi}=\int 2 D \bar{\Psi} \gamma_{5} D \Psi
$$

here the covariant differential, $D \Psi:=d \Psi+\omega \Psi$, includes the Clifford algebra valued connection one-form $\omega:=\frac{1}{4} \gamma_{a b} \omega^{a b}$. (The Dirac matrix conventions are $\gamma_{(a} \gamma_{b)}=g_{a b}, \gamma_{a b}:=\gamma_{[a} \gamma_{b]}$, $\gamma_{5}:=\gamma_{0} \gamma_{1} \gamma_{2} \gamma_{3}$. We often omit the wedge $\wedge$; for discussions of such "clifform" notation see [21 23.) This QSL satisfies the spinor-curvature identity

$$
\mathcal{L}_{\Psi}=2 D \bar{\Psi} \gamma_{5} D \Psi \equiv 2 \bar{\Psi} \Omega \gamma_{5} \Psi+d\left[(D \bar{\Psi}) \gamma_{5} \Psi+\bar{\Psi} \gamma_{5} D \Psi\right]
$$

where $\Omega=\frac{1}{4} \Omega^{a b} \gamma_{a b}=d \omega+\omega \omega$, is the Clifford algebra valued curvature 2-form. For the

special case $\Psi=\vartheta \psi$, which includes the orthonormal frame one-form $\vartheta:=\gamma_{a} \vartheta^{a}=\gamma_{a} e^{a}{ }_{\mu} d x^{\mu}$, the rhs of $(\overline{0})$ expands to

$$
\bar{\psi} \psi \Omega^{a b} \wedge \eta_{a b}+\bar{\psi} \gamma_{5} \psi \Omega_{a b} \wedge \vartheta^{a} \wedge \vartheta^{b}+d\left[D(\bar{\psi} \vartheta) \gamma_{5} \vartheta \psi+\bar{\psi} \vartheta \gamma_{5} D(\vartheta \psi)\right],
$$

where we have introduced the convenient (Hodge) dual basis $\eta^{a \cdots}:=*\left(\vartheta^{a} \wedge \cdots\right)$. For a spinor field $\psi$, normalized according to

$$
\bar{\psi} \psi=1, \quad \bar{\psi} \gamma_{5} \psi=0
$$

we get

$$
\mathcal{L}_{\psi}=2 D(\bar{\psi} \vartheta) \gamma_{5} D(\vartheta \psi) \equiv \Omega^{a b} \wedge \eta_{a b}+d\left[D(\bar{\psi} \vartheta) \gamma_{5} \vartheta \psi+\bar{\psi} \vartheta \gamma_{5} D(\vartheta \psi)\right]
$$

Since $\Omega^{a b} \wedge \eta_{a b}=-R * 1$, this QSL differs from the standard Hilbert scalar curvature Lagrangian only by an exact differential. In the action this corresponds to a boundary term which does not affect the local equations of motion [24].

\section{SPINOR GAUGE INVARIANCE OF THE QSL}

From the form of the Lagrangian (10), the QSL action for an extended region actually depends on the (normalized) spinor field only through the boundary term, not locally. A change of the spinor field within the interior of the region will leave the action unchanged. Consequently the Dirac spinor field $\psi$ has complete local gauge invariance subject to the two restrictions (9). This 6 real parameter spinor gauge freedom can be represented in the form $\psi=U \psi_{0}$ where $\psi_{0}$ is a normalized Dirac spinor with constant components and $U$ is the Dirac spinor representation of a Lorentz transformation. Thus the gauge freedom

of the normalized spinor field is a kind of local Lorentz gauge freedom. Considering the scalar curvature term in the Lagrangian (10), it can be recognized that the theory also has the usual local Lorentz gauge freedom associated with transformations of the orthonormal 
frame. Hence there appears to be two Lorentz gauge freedoms here. But are they really independent?

Considering the covariant appearance of the boundary term in (10) this seems doubtful. Usually we regard a transformation of Lorentz frame as inducing associated transformations on the components of all tensors and spinors. Under such a transformation the Lagrangian boundary term is a Lorentz invariant. But now we are contemplating independent transformations of the spinor and frame field. How does the boundary term behave?

The boundary term is

$$
\left(D \bar{\psi} \gamma_{a} \gamma_{5} \gamma_{b} \psi-\bar{\psi} \gamma_{a} \gamma_{5} \gamma_{b} D \psi\right) \vartheta^{a} \wedge \vartheta^{b}+\left(\bar{\psi} \gamma_{a} \gamma_{5} \gamma_{b} \psi-\bar{\psi} \gamma_{b} \gamma_{5} \gamma_{a} \psi\right) D \vartheta^{a} \wedge \vartheta^{b}
$$

Let us consider a gauge transformed spinor field $\psi^{\prime}=U \psi$. Then $\overline{\psi^{\prime}}=\bar{\psi} U^{-1}, D \psi^{\prime}=U D \psi$ and $D \overline{\psi^{\prime}}=D(\bar{\psi}) U^{-1}$. The gauge transformed boundary term then becomes

$$
\begin{aligned}
& \left(D \bar{\psi} U^{-1} \gamma_{a} U \gamma_{5} U^{-1} \gamma_{b} U \psi-\bar{\psi} U^{-1} \gamma_{a} U \gamma_{5} U^{-1} \gamma_{b} U D \psi\right) \vartheta^{a} \wedge \vartheta^{b} \\
& \quad+\left(\bar{\psi} U^{-1} \gamma_{a} U \gamma_{5} U^{-1} \gamma_{b} U \psi-\bar{\psi} U^{-1} \gamma_{b} U \gamma_{5} U^{-1} \gamma_{a} U \psi\right) D \vartheta^{a} \wedge \vartheta^{b} .
\end{aligned}
$$

The unitary transformations on the gammas induce Lorentz transformations, $U^{-1} \gamma_{a} U=$ $\gamma_{c} L_{a}^{c}$, on the orthonormal frame indices. Such a transformation is entirely equivalent to applying the transformation $\vartheta^{\prime c}=L_{a}^{c} \vartheta^{a}$ to the orthonormal frame alone. Hence the boundary term really has one physically independent Lorentz gauge freedom.

\section{EQUIVALENCE OF THE LAGRANGIANS}

Without losing any physics, we can confine our attention to representations where the spinor field and the orthonormal frame are tied together. A convenient choice fixing one of the Lorentz gauges is $d \psi=0$; in other words the components of $\psi$ are constant in the present frame. This locks the spinor and the orthonormal frame together. The pair then still retain the other Lorentz gauge freedom. For a general analysis there may be some advantage in regarding this condition as binding the orthonormal frame to the spinor field which (viewed as a geometric object, not as a set of components) retains its Lorentz gauge freedom. However for our immediate needs it is more perspicuous to consider the condition as tying the spinor to the orthonormal frame, with the latter still retaining its own local Lorentz gauge freedom.

We could establish the equivalence by directly expanding the Lagrangian

$$
\mathcal{L}_{\psi}=2 D(\bar{\psi} \vartheta) \gamma_{5} D(\vartheta \psi)=2(d(\bar{\psi} \vartheta)+\bar{\psi} \vartheta \omega) \gamma_{5}(d(\vartheta \psi)+\omega \vartheta \psi)
$$

using $d \psi=0$, however an indirect calculation is more efficient. We consider the boundary term on the rhs of the Lagrangian (10). With the gauge choice $d \psi=0$ in the present frame, we find

$$
\begin{aligned}
{[d(\bar{\psi} \vartheta)+\bar{\psi} \vartheta \omega] \gamma_{5} \vartheta \psi } & +\bar{\psi} \vartheta \gamma_{5}[d(\vartheta \psi)+\omega \vartheta \psi] \equiv \bar{\psi}(d \vartheta+\vartheta \omega) \gamma_{5} \vartheta \psi+\bar{\psi} \vartheta \gamma_{5}(d \vartheta+\omega \vartheta) \psi \\
& \equiv-\bar{\psi} \psi \omega^{a b} \wedge \eta_{a b}+\bar{\psi} \gamma_{5} \psi \omega_{a b} \wedge \vartheta^{a} \wedge \vartheta^{b}+\bar{\psi} \gamma_{5} \psi d \vartheta^{a} \wedge \vartheta_{a}
\end{aligned}
$$

Hence, taking into account the spinor field normalization conditions (9), the Lagrangian (10) is equivalent to 


$$
\mathcal{L} \equiv\left(d \omega^{a b}+\omega^{a}{ }_{c} \wedge \omega^{c b}\right) \wedge \eta_{a b}-d\left(\omega^{a b} \wedge \eta_{a b}\right) \equiv \omega^{a}{ }_{c} \wedge \omega^{c b} \wedge \eta_{a b}+\omega^{a b} \wedge d \vartheta^{c} \wedge \eta_{a b c} .
$$

If we vary the connection independently, we find (with no source) a relation (equivalent to vanishing torsion) which shows that the connection is a particular linear combination of $d \vartheta$. This relation is just the usual expression for the orthonormal frame (Riemannian) connection coefficients $\omega_{a b c}=\frac{1}{2}\left(C_{c a b}-C_{b c a}-C_{a b c}\right)$, where $C_{b c}^{a}:=-d \vartheta^{a}\left(e_{b}, e_{c}\right)$. Inserting these values into the rhs of (15) gives an explicit form of the Møller Lagrangian (11) for tetrad gravity:

$$
\mathcal{L}_{M}=\left(\frac{1}{4} C_{a}^{b c} C^{a}{ }_{b c}-C_{c}{ }^{c b} C^{a}{ }_{a b}+\frac{1}{2} C_{b a c} C^{a b c}\right) * 1,
$$

The equivalent covariant description in terms of teleparallel geometry (5) readily follows since the torsion 2 -form

$$
\frac{1}{2} T^{a}{ }_{\mu \nu} d x^{\mu} \wedge d x^{\nu}=T^{a}:=D \vartheta^{a}:=d \vartheta^{a}+\omega^{a}{ }_{b} \wedge \vartheta^{b},
$$

reduces, in an OT frame, to $d \vartheta^{a}$. Hence we have established an equivalence between the QSL (13) and the tetrad/teleparallel representations of GR. As we have mentioned, these latter representations were used by Møller to construct a gravitational energy-momentum density. Consequently the corresponding energy-momentum localization for the QSL and the Møller representations are also equivalent.

\section{ENERGY-MOMENTUM QUASILOCALIZATION}

It is instructive to consider this point from the QSL expressions. The energy-momentum density can be identified with the Hamiltonian. The Hamiltonian can be constructed from the action by choosing a timelike evolution vector field $N$ such that $i_{N} d t=1$ and splitting the action: $S=\int \mathcal{L}=\int d t \int i_{N} \mathcal{L}$. Applied to $\mathcal{L}_{\psi}$ this procedure leads [1] to the 4-covariant QSL Hamiltonian 3-form [25] (i.e., the Noether translation generator along $N$ )

$$
\mathcal{H}(N)=2\left[D(\bar{\psi} \not X) \gamma_{5} D(\vartheta \psi)+D(\bar{\psi} \vartheta) \gamma_{5} D(\not \mathcal{W} \psi)\right] .
$$

A notable feature of this QSL Hamiltonian is that it is already asymptotically $O\left(1 / r^{4}\right)$; consequently, its integral will be finite and its variation will have an $O\left(1 / r^{3}\right)$ boundary term which will vanish asymptotically, so there is no need for any further boundary term adjustment. In fact the Hamiltonian expression (18) could have been obtained from the usual (linear in the Einstein tensor) Hamiltonian by adding a certain total differential (although important for the value of energy-momentum such a total differential does not effect the equations of motion), as the following identity reveals:

$$
\mathcal{H}(N) \equiv 2 \bar{\psi} \psi N^{\mu} G_{\mu \nu} * \vartheta^{\nu}+2 d\left[\bar{\psi} \not X \gamma_{5} D(\vartheta \psi)+D(\bar{\psi} \vartheta) \gamma_{5} \not X \psi\right] .
$$

This identity also shows that the derivatives of $\psi$ itself are not so important - up to an exact differential $\mathcal{H}(N)$ is algebraic in $\psi$ - rather that these factors arrange for the correct quadratic connection terms.

The Hamiltonian (18) looks covariant but what does it mean physically? When the constraint equations are satisfied, the value of the Hamiltonian is given by the boundary term. In the $d \psi=0$ gauge the Hamiltonian boundary term is 


$$
\begin{aligned}
\bar{\psi} \not X \gamma_{5} D(\vartheta \psi)+D(\bar{\psi} \vartheta) \gamma_{5} \not X X & =N^{c} \bar{\psi}\left[\gamma_{c} \gamma_{5}(d \vartheta+\omega \vartheta)+(d \vartheta+\vartheta \omega) \gamma_{5} \gamma_{c}\right] \psi \\
& =-N_{c} \bar{\psi} \gamma_{5} \psi d \vartheta^{c}-N^{c} \bar{\psi} \psi \epsilon_{a b c d} \omega^{a b} \wedge \vartheta^{d} .
\end{aligned}
$$

Hence, with the normalizations (9), we find that the value of the boundary term is

$$
\omega^{a b} \wedge i_{N} \eta_{a b}=N^{c} \omega^{a b} \wedge \eta_{a b c}=N^{c} \Gamma^{a b}{ }_{d} \delta_{a b c}^{e f d} \eta_{e f},
$$

a well known expression for the superpotential [27] associated with Møller's energymomentum "tensor". This superpotential is a tensor with respect to coordinate transformations but is not tensorial with respect to the local Lorentz gauge freedom. Without some gauge fixing condition the Møller energy-momentum "tensor", and likewise the QSL formulation does not determine a well defined gravitational energy localization. Møller realized the need for some Lorentz gauge condition and even proposed one [6] (which did not prove to be so satisfactory). More recently a gauge condition has been proposed which would determine certain special orthonormal frames [28]. However, both of these conditions depend (like typical gauge conditions) on the solution of a partial differential equation; hence the gauge fixed Lorentz frames are inherently nonlocal. Using such gauge fixed frames will not yield a true local energy momentum density.

\section{DISCUSSION}

In summary, we have demonstrated that a particular quadratic spinor Lagrangian (QSL) is equivalent to the tetrad/teleparallel version of Einstein's GR theory. The corresponding energy-momentum density is equivalent to Møller's 1961 "tensor".

Our analysis raises a question. First, to what extent is our conclusion dependent on the particular choice of QSL (13)? Consider any Lagrangian, quadratic in the derivatives of some field $W$ (spinor or otherwise), which differs from the Hilbert Lagrangian only by an exact differential:

$$
\mathcal{L}_{W}=(D W)^{2} \equiv-R * 1+d(\ldots) .
$$

Then $W$ is necessarily a pure local gauge field - since the action depends on $W$ only through a boundary term. In practice we only succeeded in getting the scalar curvature in this type of identity by using spinor fields. All the spinor curvature identities [19] involving the scalar curvature (the Hilbert Lagrangian) which we found, differed from (13) only by torsion terms, which would make no contribution to the field equations in the sourceless case. (For the case with sources which couple to the Riemannian connection, we can recover Einstein's theory by suppressing the torsion using a Lagrange multiplier term.) Hence all QSLs leading to Einstein's theory are essentially equivalent to the particular one discussed here.

It is well known that the Møller energy-momentum "tensor" and the associated superpotential depends on the local Lorentz frame gauge. The total energy-momentum within a finite region actually depends only on the integral of the superpotential over the boundary - hence it depends on the local frame only through the values on the boundary. Similarly, the spinor field is purely a local gauge field; only its value on the boundary influences the calculation of energy-momentum within a region. From either representation we once again 
see energy-momentum as quasilocal [26,27], depending on the fields and the gauge choice on the boundary.

We have shown that the role of the spinor field in the QSL representation is essentially cosmetic; it allows a neat alternate version of the tetrad/teleparallel representation of GR. (Actually the representations are not quite equivalent because of the 2 to 1 relation between the spinor and frame gauge groups.) This is not to say that the QSL representation is useless. The QSLs can essentially replace an orthonormal frame with a spinor field (this works because a normalized spinor field determines an orthonormal frame up to an overall constant Lorentz transformation) which may have some advantages. One is that, like any

other new representation, the QSL suggests certain generalizations (e.g., to complex self dual representations) 29]. This present work does not preclude the possibility that a QSL would lead to a representation with genuinely new features. That could happen if it was not directly connected to the Hilbert scalar curvature Lagrangian.

\section{ACKNOWLEDGMENTS}

We would like to thank the referees for their helpful suggestions. This work was supported by the National Science Council of the R.O.C. under grant No. NSC87-2112-M-008-007, NSC88-2112-M-008-018. 


\section{REFERENCES}

[1] J. M. Nester and R. S. Tung, Gen. Rel. Grav. 27, 115-119 (1995).

[2] R. S. Tung and T. Jacobson, Class. Quantum Grav. 12, L51-55 (1995).

[3] R. S. Tung, "A vector spinor formulation for general relativity", PhD Dissertation, (National Central University, 1996, unpublished)

[4] D. C. Robinson, Class. Quantum Grav. 12, 307-315 (1996); Int. J. Theor. Phys. 37, 2067-2078 (1998).

[5] R. S. Tung, "Gravitation as a supersymmetric gauge theory", preprint (1999).

[6] C. Møller, Mat. Fys. Dan. Vid. Selsk. 1, No.10, 1-50 (1961); Ann. Phys. 12, 118-133 (1961).

[7] For the body of our work we consider the source free 'vacuum' theory. The issue of coupling to sources is noted later.

[8] J. A. Schouten, Ricci Calculus, 2nd edition (Springer-Verlag, London, 1954) p 141.

[9] Y. M. Cho, Phys. Rev. D14, 2521-2525 (1976).

[10] H. Meyer, Gen. Rel. Grav. 14, 531-547 (1982).

[11] J. M. Nester, Int. J. Mod. Phys. A 4, 1755-1772 (1989); Phys. Lett. A 139, 112-114 (1989).

[12] E. W. Mielke, Ann. Phys. 219, 78-108 (1992).

[13] J. W. Maluf, J. Math. Phys. 35, 335-343 (1994); J. Math. Phys. 36, 4242-4247 (1995); J. Math. Phys. 37, 6293-6301 (1996).

[14] F. Gronwald, Int. J. Mod. Phys. D 6, 263-303 (1997), §4.

[15] V. C. Andrade and J. G. Pereira, Phys. Rev. D 56, 4689-4695 (1997).

[16] U. Muench, F. Gronwald and F. W. Hehl, Gen. Rel. Grav. 30, 933-961 (1998).

[17] There are other teleparallel representations of GR, see J. M. Nester and J.-J. Yo, "Symmetric Teleparallel General Relativity", Chin. J. Phys. (1999) in press, (Los Alamos E-Print Archive: gr-qc/9809049). Also note that there are many teleparallel theories which are not equivalent to GR, e.g., K. Hayashi and T. Shirafuji, Phys. Rev. D 19, 3524-3553 (1979).

[18] Concerning the inclusion of sources in the field equations, the proper source coupling is such that a test particle without spin will only feel the Riemannian structure. In GR sources with spin couple to the connection; their standard GR coupling is not equivalent to 'minimal coupling' with respect to the teleparallel geometry.

[19] J. M. Nester, R. S. Tung and V. V. Zhytnikov, Class. Quantum Grav. 11, 983-987 (1994).

[20] I. Bars and S. W. MacDowell, Phys. Lett. B 71, 111-114 (1977); Gen Rel. Grav. 10, 205-209 (1979).

[21] E. W. Mielke, Geometrodynamics of Gauge Fields-on the Geometry of Yang-Mills and Gravitational Gauge Theories (Akademie, Berlin, 1987).

[22] A. Dimakis and F. Müller-Hoissen F, Class. Quantum Grav. 8, 2093-2132 (1991).

[23] F. Estabrook, Class. Quantum Grav. 8, L151-154 (1991).

[24] For the equation of motion with sources, the equivalent to the GR source coupling is reproduced by incorporating the metric (via the tetrad) into the source Lagrangian. In some cases that may be easily translated into an equivalent coupling to the spinor field $\psi$. However, we do not expect to find a general prescription for coupling the QSL spinor 
field to a source in a way that would be compatible with the spinor's local Lorentz gauge freedom.

[25] This Hamiltonian is similar to but distinct from the one associated with the Witten positive energy proof [1,26].

[26] C. M. Chen, J. M. Nester and R. S. Tung, Phys. Lett. A 203 , 5-11 (1995).

[27] C. M. Chen and J. M. Nester, "Quasilocal quantities for GR and other gravity theories", Class. Quantum Grav. (1999) in press, (Los Alamos E-Print Archive: gr-qc/9809020).

[28] J. M. Nester, J. Math Phys. 33, 910-913 (1992).

[29] Some recent works [30,31] have noted a connection between the QSL formulation and chiral supergravity. For a connection with Ashtekar's new variables see [32].

[30] E. W. Mielke and A. Macías, (Los Alamos E-Print Archive: gr-qc/9902077).

[31] A. Macías, Class. Quantum Grav. 13, 3163-3174 (1996).

[32] R. P. Wallner, J. Math. Phys. 36, 6937 (1995). 